\title{
Multiple zeta values for coordinatewise limits at non-positive integers
}

\author{
by \\ Yoshitaka Sasaki (Nagoya)
}

1. Introduction. The multiple zeta function of depth $k$ is defined by

$$
\zeta_{k}\left(s_{1}, \ldots, s_{k}\right):=\sum_{0<n_{1}<\cdots<n_{k}} \frac{1}{n_{1}^{s_{1}} \cdots n_{k}^{s_{k}}},
$$

where $s_{i}(i=1, \ldots, k)$ are complex variables. When $s_{i}(i=1, \ldots, k)$ are positive integers with $s_{k} \geq 2$, the multiple zeta function $\zeta_{k}\left(s_{1}, \ldots, s_{k}\right)$ is absolutely convergent and its values are called multiple zeta values. Euler evaluated several special cases of double zeta values. Further, he showed a very interesting formula which is the origin of the sum formula in [4]. At present, a number of relations among multiple zeta values are known, for instance, the sum formula [4], [5], Hoffman's relation [6] and Ohno's relation [11].

On the other hand, Atkinson [3] investigated the analytic properties of the double zeta function and gave the analytic continuation of $\zeta_{2}(u, v)$ to consider the mean square of the Riemann zeta function on the critical line. Atkinson's essential tool is the Euler-Maclaurin summation formula and the Poisson summation formula. Generally, the meromorphic continuation of $\zeta_{k}\left(s_{1}, \ldots, s_{k}\right)$ was given by Zhao [12] by using distribution theory, and independently by Akiyama, Egami and Tanigawa [1] by using the EulerMaclaurin summation formula. Further, Matsumoto [9] showed the meromorphic continuation of more general multiple zeta functions by using the Mellin-Barnes integral. A functional equation for the multiple zeta function which holds for any depth, similar to that for the Riemann zeta function, has not been discovered yet. However, Matsumoto [10] found a functional equation for the double zeta function.

In this article we deal with multiple zeta values at non-positive integers. Values of the Riemann zeta function at non-positive integers can be written 
by using Bernoulli numbers. Hence we are interested in similar expressions for multiple zeta values at non-positive integers. In this case, we must take care of the singularities of the multiple zeta function. The multiple zeta function $\zeta_{k}\left(s_{1}, \ldots, s_{k}\right)$ has singularities on

$$
\begin{aligned}
s_{k} & =1, \\
s_{k-1}+s_{k} & =2,1,0,-2,-4, \ldots
\end{aligned}
$$

and

$$
\sum_{i=1}^{j} s_{k-i+1} \in \mathbb{Z}_{\leq j} \quad(j=3,4, \ldots, k),
$$

where $\mathbb{Z}_{\leq \xi}$ is the set of integers less than or equal to $\xi ; \mathbb{Z}_{\geq \xi}$ is defined similarly. As can be seen from (1.3), each point $\left(s_{1}, \ldots, s_{k}\right)=\left(-r_{1}, \ldots,-r_{k}\right) \in \mathbb{Z}_{<0}^{k}$ is a singularity of the multiple zeta function. It is known that $\left(-r_{1}, \ldots,-r_{k}\right)$ is a point of indeterminacy of $\zeta_{k}\left(s_{1}, \ldots, s_{k}\right)$ (see [1]): both the local denominator and the local numerator of $\zeta_{k}\left(s_{1}, \ldots, s_{k}\right)$ vanish at $\left(-r_{1}, \ldots,-r_{k}\right)$. Therefore the values of $\zeta_{k}\left(s_{1}, \ldots, s_{k}\right)$ at $\left(-r_{1}, \ldots,-r_{k}\right)$ depend on the limiting process. In [2], Akiyama and Tanigawa gave some formulas for the regular and reverse values, which are defined by

$$
\begin{aligned}
\zeta_{k}\left(-r_{1}, \ldots,-r_{k}\right) & :=\lim _{s_{1} \rightarrow-r_{1}} \cdots \lim _{s_{k} \rightarrow-r_{k}} \zeta_{k}\left(s_{1}, \ldots, s_{k}\right), \\
\zeta_{k}^{R}\left(-r_{1}, \ldots,-r_{k}\right) & :=\lim _{s_{k} \rightarrow-r_{k}} \cdots \lim _{s_{1} \rightarrow-r_{1}} \zeta_{k}\left(s_{1}, \ldots, s_{k}\right),
\end{aligned}
$$

respectively. These values can be found by using recurrence relations as in Lemmas 3.1 and 3.2 below. Further, Akiyama and Tanigawa considered the central values given by

$$
\zeta_{k}^{C}\left(-r_{1}, \ldots,-r_{k}\right):=\lim _{\varepsilon \rightarrow 0} \zeta_{k}\left(-r_{1}+\varepsilon, \ldots,-r_{k}+\varepsilon\right) .
$$

For the central values, one could also apply Lemma 3.1 or Lemma 3.2. However, this may be difficult when the depth $k$ is large. Kamano [7] considered the multiple Hurwitz zeta function

$$
\zeta_{k}\left(s_{1}, \ldots, s_{k} ; a\right):=\sum_{\substack{0 \leq m_{1}<\cdots<m_{k} \\ m_{i} \in \mathbb{Z}}} \frac{1}{\left(m_{1}+a\right)^{s_{1}} \cdots\left(m_{k}+a\right)^{s_{k}}} \quad\left(a \in \mathbb{R}_{>0}\right)
$$

and gave formulas for its regular values and reverse values. Further, he considered the special case of $\zeta_{k}(s ; a):=\zeta_{k}(s, \ldots, s ; a)$ and gave formulas for the central values $\zeta_{k}^{C}(-r ; a)$ for any depth $k$ and any non-negative integer $r$.

In this article, we consider all coordinatewise limits for any depth. Except regular and reverse values, such limits were not treated in $[1,2]$. Some interesting relations among those values support the existence of a functional equation for the multiple zeta function, similar to that for the Riemann zeta function. 
First we consider all coordinatewise limits for the case of depth 3 . This case is important, since the multiple zeta function of depth $k$ is constructed from that of depth $k-1$ inductively (see Lemmas 3.1 and 3.2). Secondly, we try to generalize those results. However, it is difficult to give an explicit formula for multiple zeta values for all coordinatewise limits in the case of depth $k \geq 4$, since already when the depth is 4 there exist 24 kinds of limiting processes. Hence we give an algorithm to calculate multiple zeta values for all coordinatewise limits in the last section.

When the depth is 2, there are only two coordinatewise limits, namely regular and reverse values given by

$$
\begin{aligned}
\zeta_{2}\left(-r_{1},-r_{2}\right) & =\sum_{q=-1}^{r_{2}}\left(-r_{2}\right)_{q}^{+} a_{q} \zeta\left(-r_{1}-r_{2}+q\right), \\
\zeta_{2}^{R}\left(-r_{1},-r_{2}\right) & =-\sum_{q=-1}^{r_{1}}\left(-r_{1}\right)_{q}^{-} a_{q} \zeta\left(-r_{1}-r_{2}+q\right)+\zeta\left(-r_{1}\right) \zeta\left(-r_{2}\right),
\end{aligned}
$$

respectively (see $[1,2])$. Here

$$
(s)_{q}^{ \pm}:= \begin{cases}s(s+1) \cdots(s+q-1) & \text { if } q=1,2, \ldots, \\ \pm 1 & \text { if } q=0, \\ 1 /(s-1) & \text { if } q=-1,\end{cases}
$$

$a_{q}:=B_{q+1} /(q+1)$ ! and $B_{n}$ is the $n$th Bernoulli number. Therefore our starting point is the case of depth 3 .

We introduce the following notation. Let $\left\{i_{1}, \ldots, i_{k}\right\}=\{1, \ldots, k\}$ and define

$$
\zeta_{k}\left(-i_{1} r_{1}, \ldots,-i_{k} r_{k}\right):=\lim _{\substack{s_{j} \rightarrow-r_{j} \\ i_{j}=k}} \cdots \lim _{\substack{s_{j} \rightarrow-r_{j} \\ i_{j}=1}} \zeta_{k}\left(s_{1}, \ldots, s_{k}\right) .
$$

For instance, $\zeta_{3}\left(\stackrel{3}{-} r_{1},-{ }_{2}, \stackrel{1}{2},-r_{3}\right)$ and $\zeta_{3}\left(-r_{1},-2 r_{2},-r_{3}\right)$ are the regular value and the reverse value, respectively. In Section 6, we shall prove some relations among multiple zeta values of depth 3 for all coordinatewise limits. For instance, we show that

$$
\begin{aligned}
& \zeta_{3}(\stackrel{1}{-1}, \stackrel{2}{-2}, \stackrel{3}{-}-3)-\zeta_{3}(\stackrel{1}{-1}, \stackrel{3}{-}-2, \stackrel{2}{-3})-\zeta_{3}(\stackrel{2}{-1}, \stackrel{1}{-2}, \stackrel{3}{-3})+\zeta_{3}(\stackrel{2}{-1}, \stackrel{3}{-2}, \stackrel{1}{-3}) \\
& +\zeta_{3}(\stackrel{3}{-1}, \stackrel{1}{-2}, \stackrel{2}{-3})-\zeta_{3}(\stackrel{3}{-1}, \stackrel{2}{-2}, \stackrel{1}{-3})=0
\end{aligned}
$$

(see also Example 2.5 below). Further, in Section 7, we show that a generalization of Corollary 2.2 and the second assertion of Proposition 6.4 below hold for any depth. In the last section, we give an algorithm to calculate multiple zeta values of depth $k$ for all coordinatewise limits. 
Recently, Komori [8] treated more general multiple zeta functions and obtained an integral representation for them. He calculated several numerical values of those multiple zeta functions at non-positive integers. In the next section, we compute some numerical values in Example 2.5. They coincide with those obtained by Komori.

\section{Formulas for special values when the depth is 3}

ThEOREM 2.1. For any non-negative integers $r_{i}(i=1,2,3)$, we have

$$
\begin{aligned}
\zeta_{3}\left(\stackrel{2}{-} r_{1},-{ }^{3} r_{2},-r_{3}\right)= & \sum_{q=-1}^{r_{3}}\left(-r_{3}\right)_{q}^{+} a_{q} \zeta_{2}^{R}\left(-r_{1},-r_{2}-r_{3}+q\right), \\
\zeta_{3}\left(\stackrel{1}{-} r_{1}, \stackrel{3}{-} r_{2}, \stackrel{2}{-} r_{3}\right)= & -\sum_{q=-1}^{r_{1}}\left(-r_{1}\right)_{q}^{-} a_{q} \zeta_{2}\left(-r_{1}-r_{2}+q,-r_{3}\right) \\
& +\zeta\left(-r_{1}\right) \zeta_{2}\left(-r_{2},-r_{3}\right),
\end{aligned}
$$

where $\zeta_{2}$ and $\zeta_{2}^{R}$ are regular values (1.4) and reverse values (1.5), respectively.

Corollary 2.2 .

$$
\zeta_{3}\left(\stackrel{2}{-} r_{1}, \stackrel{3}{-} r_{2},-\stackrel{1}{-} r_{3}\right)=\zeta_{3}\left(\stackrel{1}{-} r_{1},-\stackrel{3}{-} r_{2}, \stackrel{2}{-} r_{3}\right) .
$$

Theorem 2.3. For any non-negative integers $r_{i}(i=1,2,3)$, we have

$$
\begin{aligned}
& \zeta_{3}\left(\stackrel{3}{-} r_{1}, \stackrel{1}{-} r_{2},-r_{3}\right)=Z_{3}-(-1)^{r_{1}} r_{1} ! \sum_{q=-1}^{r_{3}}\left(-r_{3}\right)_{q}^{+} a_{q} a_{r_{1}+r_{2}+r_{3}+1-q}\left(r_{2}+r_{3}-q\right) \text { ! } \\
& +(-1)^{r_{3}} r_{2} ! r_{3} ! \zeta\left(-r_{1}\right) a_{r_{2}+r_{3}+1}, \\
& \zeta_{3}\left(\stackrel{2}{-} r_{1},-r_{2},-r_{3}\right)=Z_{3}-(-1)^{r_{3}} r_{3} ! \sum_{q=-1}^{r_{1}}\left(-r_{1}\right)_{q}^{-} a_{q} a_{r_{1}+r_{2}+r_{3}+1-q}\left(r_{1}+r_{2}-q\right) !
\end{aligned}
$$

where

$$
\begin{aligned}
Z_{3}= & -\sum_{q=-1}^{r_{3}}\left(-r_{3}\right)_{q}^{+} a_{q} \sum_{p=-1}^{r_{1}}\left(-r_{1}\right)_{p}^{-} a_{p} \zeta\left(-r_{1}-r_{2}-r_{3}+p+q\right) \\
& +\zeta\left(-r_{1}\right) \sum_{q=-1}^{r_{3}}\left(-r_{3}\right)_{q}^{+} a_{q} \zeta\left(-r_{2}-r_{3}+q\right) .
\end{aligned}
$$

REMARK 2.4. The value of $Z_{3}$ equals the value given in Corollary 2.2. This follows from (4.1) and (4.2) below. 
EXAMPLE 2.5. When $s_{1}=s_{2}=s_{3}=0$,

$$
\begin{aligned}
& \zeta_{3}(\stackrel{3}{0}, \stackrel{2}{0}, \stackrel{1}{0})=-\frac{1}{4}, \quad \zeta_{3}(\stackrel{1}{0}, \stackrel{2}{0}, \stackrel{3}{0})=-\frac{3}{8}, \\
& \zeta_{3}(\stackrel{2}{0} \stackrel{3}{0} \stackrel{1}{0}, 0)=\zeta_{3}(\stackrel{1}{0}, \stackrel{3}{0}, \stackrel{2}{0})=-\frac{7}{24}, \\
& \zeta_{3}(\stackrel{3}{0} \stackrel{2}{0} \stackrel{2}{0})=-\frac{7}{24}, \quad \zeta_{3}(\stackrel{2}{0}, \stackrel{3}{0}, \stackrel{3}{0})=-\frac{3}{8} .
\end{aligned}
$$

When $s_{1}=-1, s_{2}=-2, s_{3}=-3$,

$$
\begin{aligned}
& \zeta_{3}(\stackrel{3}{-1}, \stackrel{2}{-2}, \stackrel{1}{-3})=-\frac{101}{100800}, \quad \zeta_{3}(\stackrel{1}{-1}, \stackrel{2}{-2}, \stackrel{3}{-3})=-\frac{43}{40320}, \\
& \zeta_{3}(\stackrel{2}{-1}, \stackrel{3}{-2}, \stackrel{1}{-3})=\zeta_{3}(\stackrel{1}{-1}, \stackrel{3}{-2}, \stackrel{2}{-3})=-\frac{53}{50400}, \\
& \zeta_{3}(\stackrel{3}{-} \underset{-1}{-1}-\underset{2}{2}, \stackrel{2}{-3})=-\frac{101}{100800}, \quad \zeta_{3}(\stackrel{2}{-1}, \stackrel{1}{-2}, \stackrel{3}{-3})=-\frac{43}{40320} .
\end{aligned}
$$

When $s_{1}=-2, s_{2}=-4, s_{3}=-6$,

$$
\begin{aligned}
& \zeta_{3}(\stackrel{3}{-2}, \stackrel{2}{-}-4, \stackrel{1}{-6})=\frac{105353}{8648640}, \quad \zeta_{3}(\stackrel{1}{-2}, \stackrel{2}{-4}, \stackrel{3}{-6})=\frac{104903}{8648640}, \\
& \zeta_{3}(\stackrel{2}{-2}, \stackrel{3}{-4} \stackrel{-1}{-6})=\zeta_{3}(\stackrel{1}{-} 2, \stackrel{3}{-} \underset{3}{-}, \stackrel{2}{-6})=\frac{104933}{8648640}, \\
& \zeta_{3}(\stackrel{3}{-2}, \stackrel{1}{-4}, \stackrel{2}{-6} 6)=\frac{105353}{8648640}, \quad \zeta_{3}(\stackrel{2}{-2}, \stackrel{1}{-4}, \stackrel{3}{-6})=\frac{104903}{8648640} .
\end{aligned}
$$

3. Analytic continuation. In this section and the next two, we prove the theorems of the preceding section. We follow the method of analytic continuation applied by Akiyama, Egami and Tanigawa.

Let

$$
\phi_{l}(m, s)=\frac{(s)_{l+1}^{ \pm}}{(l+1) !} \int_{m}^{\infty} \widetilde{B}_{l+1}(x) x^{-s-l-2} d x
$$

where $\widetilde{B}_{n}(x)=B_{n}(x-[x])$ and $B_{n}(x)$ is the $n$th Bernoulli polynomial. It is easily seen that

$$
\phi_{l}(m, s)=O\left(\left|(s)_{l+1}^{ \pm}\right| m^{-\Re s-l-1}\right) .
$$

We note that $\phi_{l}(m, s)=0$ for $s=0,-1, \ldots,-l$. The following lemmas hold.

Lemma 3.1 (regular type, Akiyama, Egami and Tanigawa [1]). For $\Re s_{i}>1$ $(i=1, \ldots, k)$ and any positive integer $l$, we have

$$
\begin{aligned}
\zeta_{k}\left(s_{1}, \ldots, s_{k-1}, s_{k}\right)= & \sum_{q=-1}^{l}\left(s_{k}\right)_{q}^{+} a_{q} \zeta_{k-1}\left(s_{1}, \ldots, s_{k-2}, s_{k-1}+s_{k}+q\right) \\
& -\sum_{0<n_{1}<\cdots<n_{k-1}} \frac{\phi_{l}\left(n_{k-1}, s_{k}\right)}{n_{1}^{s_{1}} \cdots n_{k-1}^{s_{k-1}}}
\end{aligned}
$$

and the last sum on the right-hand side of (3.1) is absolutely convergent 
when

$$
l>-\Re s_{k-1}-\Re s_{k}+k-2-\sum_{\substack{1 \leq i \leq k-2 \\ \Re s_{i} \leq 0}} \Re s_{i} .
$$

Lemma 3.2 (reverse type, Akiyama and Tanigawa [2]). For $\Re s_{i}>1$ $(i=1, \ldots, k)$ and any positive integer $l$, we have

$$
\begin{aligned}
\zeta_{k}\left(s_{1}, s_{2}, \ldots, s_{k}\right)= & -\sum_{q=-1}^{l}\left(s_{1}\right)_{q}^{-} a_{q} \zeta_{k-1}\left(s_{1}+s_{2}+q, s_{3}, \ldots, s_{k}\right) \\
& +\zeta\left(s_{1}\right) \zeta_{k-1}\left(s_{2}, \ldots, s_{k}\right) \\
& +\sum_{j=2}^{k-1}(-1)^{j} \zeta_{k-j}\left(s_{j+1}, \ldots, s_{k-1}, s_{k}\right) \Phi_{j}^{l}\left(s_{1}, \ldots, s_{j}\right) \\
& +(-1)^{k} \Phi_{k}^{l}\left(s_{1}, \ldots, s_{k}\right)
\end{aligned}
$$

where

$$
\Phi_{m}^{l}\left(s_{1}, \ldots, s_{m}\right):=\sum_{n_{m} \leq \cdots \leq n_{2}} \frac{\phi_{l}\left(n_{2}, s_{1}\right)}{n_{m}^{s_{m}} \cdots n_{2}^{s_{2}}} \quad(2 \leq m \leq k)
$$

and $\Phi_{m}^{l}\left(s_{1}, \ldots, s_{m}\right)$ is absolutely convergent when

$$
l>-\Re s_{1}-\Re s_{2}+m-2-\sum_{\substack{3 \leq i \leq m \\ \Re s_{i}<0}} \Re s_{i} .
$$

Note that the last sum above is 0 when $m=2$.

From Lemmas 3.1 and 3.2, since $l$ can be chosen arbitrarily large, we find inductively that the multiple zeta function can be continued to the whole $\mathbb{C}^{k}$ as a meromorphic function in $s_{1}, \ldots, s_{k}$.

4. Proof of Theorem 2.1 and Corollary 2.2. Since $(-r)_{q}^{ \pm}=0$ when $q>r$ and $r$ is a non-negative integer, we get

$$
\zeta_{3}\left(s_{1}, s_{2},-r_{3}\right)=\sum_{q=-1}^{r_{3}}\left(-r_{3}\right)_{q}^{+} a_{q} \zeta_{2}\left(s_{1}, s_{2}-r_{3}+q\right)
$$

by Lemma 3.1. The right-hand side of the above formula is a finite sum of multiple zeta values of depth 2 . Hence, from the definition of reverse values, we see that the first assertion of Theorem 2.1 holds.

Similarly, by using Lemma 3.2, we can get the second assertion of Theorem 2.1.

To prove Corollary 2.2, we use the recurrence relation (1.5). Substituting (1.5) into the right-hand side of (2.1), we have 


$$
\begin{aligned}
& \zeta_{3}\left(-r_{1}^{2}, \stackrel{3}{3} r_{2},-r_{3}\right) \\
& =-\sum_{q=-1}^{r_{3}}\left(-r_{3}\right)_{q}^{+} a_{q} \sum_{p=-1}^{r_{1}}\left(-r_{1}\right)_{p}^{-} a_{p} \zeta\left(-r_{1}-r_{2}-r_{3}+p+q\right) \\
& \quad+\zeta\left(-r_{1}\right) \sum_{q=-1}^{r_{3}}\left(-r_{3}\right)_{q}^{+} a_{q} \zeta\left(-r_{2}-r_{3}+q\right) .
\end{aligned}
$$

On the other hand, substituting (1.4) into the right-hand side of (2.2), we have

$$
\begin{aligned}
& \zeta_{3}\left(\begin{array}{c}
1 \\
-r_{1},
\end{array} \stackrel{3}{3} r_{2},-r_{3}\right) \\
& =-\sum_{q=-1}^{r_{1}}\left(-r_{1}\right)_{q}^{-} a_{q} \sum_{p=-1}^{r_{3}}\left(-r_{3}\right)_{p}^{+} a_{p} \zeta\left(-r_{1}-r_{2}-r_{3}+p+q\right) \\
& \quad+\zeta\left(-r_{1}\right) \sum_{q=-1}^{r_{3}}\left(-r_{3}\right)_{q}^{+} a_{q} \zeta\left(-r_{2}-r_{3}+q\right) .
\end{aligned}
$$

Hence we obtain Corollary 2.2.

5. Proof of Theorem 2.3. From Lemmas 3.1 and 3.2, we have

$$
\begin{aligned}
\zeta_{3}\left(s_{1},-r_{2}, s_{3}\right)= & -\sum_{q=-1}^{l}\left(s_{3}\right)_{q}^{+} a_{q} \sum_{p=-1}^{l^{\prime}}\left(s_{1}\right)_{p}^{-} a_{p} \zeta\left(s_{1}-r_{2}+s_{3}+p+q\right) \\
& +\zeta\left(s_{1}\right) \sum_{q=-1}^{l}\left(s_{3}\right)_{q}^{+} a_{q} \zeta\left(-r_{2}+s_{3}+q\right) \\
& +\sum_{q=-1}^{l}\left(s_{3}\right)_{q}^{+} a_{q}\left\{\zeta\left(s_{3}\right) \Phi_{2}^{l^{\prime}}\left(s_{1},-r_{2}\right)-\Phi_{3}^{l^{\prime}}\left(s_{1},-r_{2}, s_{3}\right)\right\} \\
& -\sum_{0<n_{1}<n_{2}} \frac{\phi_{l}\left(n_{2}, s_{3}\right)}{n_{1}^{s_{1}} n_{2}^{-r_{2}}},
\end{aligned}
$$

where $l$ and $l^{\prime}$ are arbitrarily large positive integers. Then the series $\Phi_{i}^{l^{\prime}}$ and the last sum above are absolutely convergent. Further, these terms vanish as $s_{3} \rightarrow-r_{3}$ and $s_{1} \rightarrow-r_{1}$. Thus

$$
\begin{aligned}
\zeta_{3}\left(-{ }^{3} r_{1},-r_{2}, \stackrel{2}{2} r_{3}\right)= & -\sum_{q=-1}^{r_{3}}\left(-r_{3}\right)_{q}^{+} a_{q} \sum_{p=-1}^{r_{1}}\left(-r_{1}\right)_{p}^{-} a_{p} \zeta\left(-r_{1}-r_{2}-r_{3}+p+q\right) \\
& +\zeta\left(-r_{1}\right) \sum_{q=-1}^{r_{3}}\left(-r_{3}\right)_{q}^{+} a_{q} \zeta\left(-r_{2}-r_{3}+q\right)+L_{1}+L_{2}
\end{aligned}
$$


where

$$
\begin{aligned}
& L_{1}=-\lim _{s_{1} \rightarrow-r_{1}} \sum_{q=-1}^{r_{3}}\left(-r_{3}\right)_{q}^{+} a_{q} \sum_{p=r_{1}+1}^{l^{\prime}}\left(s_{1}\right)_{p}^{-} a_{p} \zeta\left(s_{1}-r_{2}-r_{3}+p+q\right), \\
& L_{2}=\zeta\left(-r_{1}\right) \lim _{s_{3} \rightarrow-r_{3}} \sum_{q=r_{3}+1}^{l}\left(s_{3}\right)_{q}^{+} a_{q} \zeta\left(-r_{2}+s_{3}+q\right) .
\end{aligned}
$$

When $p=r_{1}+r_{2}+r_{3}+1-q$, we note that $\left(s_{1}\right)_{r_{1}+r_{2}+r_{3}+1-q}^{-}$has simple zeros at $s_{1}=0,-1, \ldots,-r_{1}-r_{2}-r_{3}+q$ and $\zeta\left(s_{1}+r_{1}+1\right)$ has a simple pole at $s_{1}=-r_{1}$ in the sum of $L_{1}$, since $l^{\prime}$ is an arbitrarily large positive integer. Therefore we obtain

$$
L_{1}=-(-1)^{r_{1}} r_{1} ! \sum_{q=-1}^{r_{3}}\left(-r_{3}\right)_{q}^{+} a_{q} a_{r_{1}+r_{2}+r_{3}+1-q}\left(r_{2}+r_{3}-q\right) ! .
$$

Similarly,

$$
L_{2}=(-1)^{r_{3}} \zeta\left(-r_{1}\right) r_{2} ! r_{3} ! a_{r_{2}+r_{3}+1} .
$$

Hence the first assertion of Theorem 2.3 holds.

Similarly, we can obtain the second assertion.

6. Relations among multiple zeta values of depth 3 . In this section, we consider relations among the values given by Theorem 2.1, the values given by Theorem 2.3, regular values, and reverse values. The starting point is the following lemma which is an essential tool in this section.

Lemma 6.1. For any non-negative integers $r_{i}(i=1,2)$, we have

$$
\zeta_{2}^{R}\left(-r_{1},-r_{2}\right)=\zeta_{2}\left(-r_{1},-r_{2}\right)+(-1)^{\mu} r_{1} ! r_{2} ! a_{r_{1}+r_{2}+1},
$$

where $\mu=r_{1}$ or $r_{2}$.

Proof. From Lemma 3.1, we obtain

$$
\lim _{s_{1} \rightarrow-r_{1}} \zeta_{2}\left(s_{1}, s_{2}\right)=\sum_{q=-1}^{l}\left(s_{2}\right)_{q}^{+} a_{q} \zeta\left(-r_{1}+s_{2}+q\right)-\sum_{n_{1}>0} \frac{\phi_{l}\left(n_{1}, s_{2}\right)}{n_{1}^{-r_{1}}} .
$$

When $q=r_{1}+r_{2}+1$, we note that $\left(s_{2}\right)_{r_{1}+r_{2}+1}^{+}$has simple zeros at $s_{2}=$ $0,-1, \ldots,-r_{1}-r_{2}$ and $\zeta\left(s_{2}+r_{2}+1\right)$ has a simple pole at $s_{2}=-r_{2}$, since $l$ is an arbitrarily large positive integer. Therefore

$$
\lim _{s_{2} \rightarrow-r_{2}}\left(s_{2}\right)_{r_{1}+r_{2}+1}^{+} \zeta\left(s_{2}-r_{2}+1\right)=(-1)^{r_{2}} r_{1} ! r_{2} !
$$


Hence we obtain

$$
\begin{aligned}
\lim _{s_{2} \rightarrow-r_{2}} \zeta_{2} & \left(-r_{1}, s_{2}\right) \\
& =\sum_{q=-1}^{r_{2}}\left(-r_{2}\right)_{q}^{+} a_{q} \zeta\left(-r_{1}-r_{2}+q\right)+(-1)^{r_{2}} r_{1} ! r_{2} ! a_{r_{1}+r_{2}+1} \\
& =\zeta_{2}\left(-r_{1},-r_{2}\right)+(-1)^{r_{2}} r_{1} ! r_{2} ! a_{r_{1}+r_{2}+1},
\end{aligned}
$$

since $\phi_{l}\left(n_{1},-r_{2}\right)=0$ and by (1.4). On the other hand, from Lemma 3.2,

$$
\zeta_{2}\left(-r_{1},-r_{2}\right)=\zeta_{2}^{R}\left(-r_{1},-r_{2}\right)-(-1)^{r_{1}} r_{1} ! r_{2} ! a_{r_{1}+r_{2}+1} .
$$

A difference between (6.2) and (6.3) is the oscillating factors $(-1)^{r_{2}}$ and $(-1)^{r_{1}}$. However, this difference does not cause a problem, since if $r_{1}+r_{2}$ is odd, then $a_{r_{1}+r_{2}+1}=0$ holds by definition.

Proposition 6.2. For any non-negative integers $r_{i}(i=1,2,3)$, we have

$$
\begin{aligned}
& \zeta_{3}\left(\stackrel{2}{-} r_{1}, \stackrel{3}{-} r_{2}, \stackrel{1}{-} r_{3}\right)=\zeta_{3}\left(\stackrel{3}{-} r_{1}, \stackrel{2}{-} r_{2},-\stackrel{1}{-} r_{3}\right) \\
& +(-1)^{r_{1}} r_{1} ! \sum_{q=-1}^{r_{3}}\left(-r_{3}\right)_{q}^{+} a_{q} a_{r_{1}+r_{2}+r_{3}+1-q}\left(r_{2}+r_{3}-q\right) !, \\
& \zeta_{3}\left(\stackrel{3}{-} r_{1}, \stackrel{1}{-} \underset{r_{2}}{ }, \stackrel{2}{-} r_{3}\right)=\zeta_{3}\left(\stackrel{3}{-} \underset{r_{1}}{ }, \stackrel{2}{-r_{2}}, \stackrel{1}{-} r_{3}\right)+(-1)^{r_{3}} r_{2} ! r_{3} ! \zeta\left(-r_{1}\right) a_{r_{2}+r_{3}+1}
\end{aligned}
$$

REMARK 6.3. Formula (6.5) expresses the difference between $\zeta_{3}\left(\begin{array}{lll}3 & 1 & * \\ * & * & *\end{array}\right)$ and $\zeta_{3}(\stackrel{3}{*}, \underset{*}{2}, *)$. In fact, from $(6.4), \zeta_{3}(\stackrel{3}{*}, * \underset{*}{1}, *)$ can be written by using $\zeta_{3}\left(\begin{array}{ccc}2 & 3 & 1 \\ * & * & *\end{array}\right)$. However, the expression of $(6.5)$ is more concise.

Proof of Proposition 6.2. Applying Lemma 6.1 with $\mu=r_{1}$ to (2.1), we have

$$
\begin{aligned}
& \zeta_{3}\left(\begin{array}{c}
2 \\
-r_{1}
\end{array}, \stackrel{3}{-} r_{2},-{ }^{1} r_{3}\right) \\
& =\sum_{q=-1}^{r_{3}}\left(-r_{3}\right)_{q}^{+} a_{q}\left\{\zeta_{2}\left(-r_{1},-r_{2}-r_{3}+q\right)\right. \\
& \left.+(-1)^{r_{1}} a_{r_{1}+r_{2}+r_{3}+1-q} r_{1} !\left(r_{2}+r_{3}-q\right) !\right\} \\
& =\zeta_{3}\left(\stackrel{3}{-} r_{1}, \stackrel{2}{-} r_{2}, \stackrel{1}{-} r_{3}\right)+(-1)^{r_{1}} r_{1} ! \sum_{q=-1}^{r_{3}}\left(-r_{3}\right)_{q}^{+} a_{q} a_{r_{1}+r_{2}+r_{3}+1-q}\left(r_{2}+r_{3}-q\right) \text { !. }
\end{aligned}
$$

Here we have used the representation of regular values of depth 3 given by Lemma 3.1. This yields the first assertion of Proposition 6.2. 
Next we prove (6.5). By combining (6.4) with (4.1), we have

$$
\begin{aligned}
\zeta_{3}\left(-r_{1},-r_{2},-r_{3}\right)= & -\sum_{q=-1}^{r_{3}}\left(-r_{3}\right)_{q}^{+} a_{q} \sum_{p=-1}^{r_{1}}\left(-r_{1}\right)_{p}^{-} a_{p} \zeta\left(-r_{1}-r_{2}-r_{3}+p+q\right) \\
& +\zeta\left(-r_{1}\right) \sum_{q=-1}^{r_{3}}\left(-r_{3}\right)_{q}^{+} a_{q} \zeta\left(-r_{2}-r_{3}+q\right) \\
& -(-1)^{r_{1}} r_{1} ! \sum_{q=-1}^{r_{3}}\left(-r_{3}\right)_{q}^{+} a_{q} a_{r_{1}+r_{2}+r_{3}+1-q}\left(r_{2}+r_{3}-q\right) !
\end{aligned}
$$

By comparing the above formula with Theorem 2.3, we obtain (6.5).

Similarly, we obtain the following proposition.

Proposition 6.4. For any non-negative integers $r_{i}(i=1,2,3)$, we have

$$
\begin{aligned}
& \zeta_{3}\left(\stackrel{1}{-} r_{1}, \stackrel{3}{-r_{2}}, \stackrel{2}{-r_{3}}\right)=\zeta_{3}\left(\stackrel{1}{-r_{1}}, \stackrel{2}{-} r_{2},-\stackrel{3}{-r_{3}}\right) \\
& +(-1)^{r_{3}} r_{3} ! \sum_{q=-1}^{r_{1}}\left(-r_{1}\right)_{q}^{-} a_{q} a_{r_{1}+r_{2}+r_{3}+1-q}\left(r_{1}+r_{2}-q\right) !, \\
& \zeta_{3}\left(\stackrel{2}{-} r_{1}, \stackrel{1}{-} r_{2}, \stackrel{3}{-} r_{3}\right)=\zeta_{3}\left(\stackrel{1}{-} r_{1}, \stackrel{2}{-} r_{2}, \stackrel{3}{-} r_{3}\right) .
\end{aligned}
$$

REMARK 6.5. Propositions 6.2 and 6.4 imply that $\zeta_{3}(*, * 2, *$ ) (which coincides with $\zeta_{3}(*, *, *)$ by Corollary 2.2$)$ is a kind of link between regular values and reverse values.

Let $\mathfrak{S}_{n}$ denote the symmetric group on $n$ symbols. Corollary 2.2 and Propositions 6.2 and 6.4 yield the following theorem.

Theorem 6.6. If $r_{1} \equiv 0(\bmod 2)$ or $r_{2}+r_{3} \equiv 1(\bmod 2)$, then

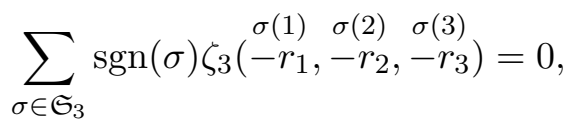

where $\operatorname{sgn}(\sigma)$ is the signature of $\sigma$.

REMARK 6.7. When $r_{1}+r_{2} \equiv 1(\bmod 2)$, we see that

$$
\sum_{\sigma \in \mathfrak{S}_{2}} \operatorname{sgn}(\sigma) \zeta_{2}\left(\begin{array}{ll}
\sigma(1) & \sigma(2) \\
\left.-r_{1},-r_{2}\right) & =0
\end{array}\right.
$$

from Lemma 6.1.

7. Generalization. In this section, we generalize Corollary 2.2 and the second assertion of Proposition 6.4. 
Recall that when $k=3$, Corollary 2.2 asserts that

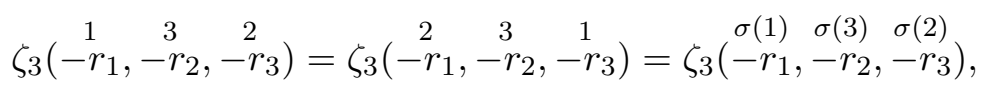

where $\sigma=\left(\begin{array}{ll}1 & 2\end{array}\right) \in \mathfrak{S}_{3}$. Generally, by induction on $k$, we have

THEOREM 7.1. Let $k$ be an odd integer satisfying $k \geq 3$. Then for any non-negative integers $r_{i}(i=1, \ldots, k)$, we have

$$
\begin{aligned}
& \zeta_{k}\left(-{ }^{1} r_{1},-\stackrel{3}{r}_{2}, \ldots,-\stackrel{k}{k}-r_{(k+1) / 2}, \ldots,-r_{k-1},-r_{k}\right)
\end{aligned}
$$

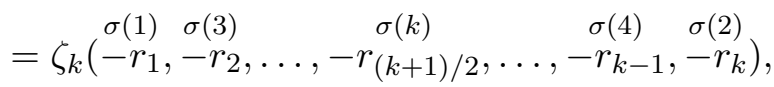

where $\sigma \in \mathfrak{S}_{k}$ is a product $\left(l_{1} l_{1}+1\right) \cdots\left(l_{n} l_{n}+1\right)$ of transpositions, with each $l_{j}$ being an odd integer satisfying $0<l_{j}<k$ and $n \leq(k-1) / 2$.

Proof. When $k=3$, the assertion has already been shown as Corollary 2.2. Next, we assume that the assertion holds for $k \leq m-2$ (where $m>5$ is an odd integer). Then from Lemmas 3.1 and 3.2, we have

$$
\begin{aligned}
& \zeta_{m}\left(-r_{1}, s_{2}, \ldots, s_{(m+1) / 2}, \ldots, s_{m-1},-r_{m}\right) \\
&=- \sum_{q=-1}^{r_{1}}\left(-r_{1}\right)_{q}^{-} a_{q} \sum_{p=-1}^{r_{m}}\left(-r_{m}\right)_{p}^{+} a_{p} \\
& \times \zeta_{m-2}\left(-r_{1}+s_{2}+q, \ldots, s_{(m+1) / 2}, \ldots, s_{m-1}-r_{m}+p\right) \\
&+\zeta\left(-r_{1}\right) \sum_{p=-1}^{r_{m}}\left(-r_{m}\right)_{p}^{+} a_{p} \zeta_{m-2}\left(s_{2}, \ldots, s_{(m+1) / 2}, \ldots, s_{m-1}-r_{m}+p\right) \\
&= \zeta_{m}\left(-r_{1}, s_{2}, \ldots, s_{(m+1) / 2}, \ldots, s_{m-1},-r_{m}\right) .
\end{aligned}
$$

The second and third lines of the above formula are finite sums of multiple zeta values of depth $m-2$. Hence, by the inductive assumption, we obtain Theorem 7.1.

THEOREM 7.2. Let $k$ be an even integer satisfying $k \geq 4$ and each $r_{i}$ $(i=1, \ldots, k)$ be a non-negative integer. Then

$$
\begin{aligned}
& \zeta_{k}\left(-\stackrel{1}{r}_{1}, \stackrel{2}{-} r_{2},-\stackrel{4}{-} r_{3}, \ldots, \underset{k}{k},-r_{(k+2) / 2}, \ldots,-r_{k-1}^{5},-r_{k}\right)
\end{aligned}
$$

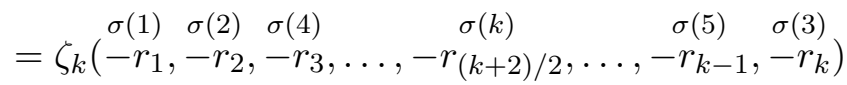

and

$$
\begin{aligned}
& \zeta_{k}\left(\stackrel{3}{-} r_{1},-r_{2}, \ldots,-\stackrel{k}{k}, \stackrel{4}{4} \underset{(k-2) / 2}{2}, \ldots,-r_{k-2}^{2},-r_{k-1},-r_{k}\right)
\end{aligned}
$$

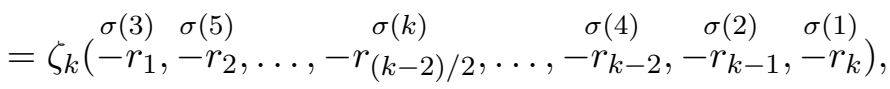


where $\sigma \in \mathfrak{S}_{k}$ is a product $\left(l_{1} l_{1}+1\right) \cdots\left(l_{n} l_{n}+1\right)$ of transpositions, with each $l_{j}$ being an even integer satisfying $0<l_{j}<k$ and $n \leq(k-2) / 2$.

Proof. From Lemma 3.2, we have

$$
\begin{aligned}
& \zeta_{k}\left(-r_{1}, s_{2}, \ldots, s_{(k+2) / 2}, \ldots, s_{k-1}, s_{k}\right) \\
& \quad=-\sum_{q=-1}^{r_{1}}\left(-r_{1}\right)_{q}^{-} a_{q} \zeta_{k-1}\left(-r_{1}+s_{2}+q, \ldots, s_{(k+2) / 2}, \ldots, s_{k-1}, s_{k}\right) \\
& \quad+\zeta\left(-r_{1}\right) \zeta_{k-1}\left(s_{2}, \ldots, s_{(k+2) / 2}, \ldots, s_{k}\right) .
\end{aligned}
$$

The right-hand side is a finite sum of multiple zeta values of depth $k-1$. Hence the first assertion of Theorem 7.2 follows from Theorem 7.1, since $k$ is an even integer.

Similarly, we obtain the second assertion by using Lemma 3.1 and Theorem 7.1.

The second assertion of Proposition 6.4 implies that if we perform $s_{3} \rightarrow$ $-r_{3}$ last, then all of those values, namely the values $\zeta_{3}\left(-r_{1},-{ }^{*}, \stackrel{3}{2},-r_{3}\right)$, are equal to each other. The same phenomenon also appears for any depth $k \geq 3$. To see this, we use the well-known formula

$$
\sum_{m=1}^{n-1} m^{r}= \begin{cases}\sum_{j=0}^{r}\left(\begin{array}{l}
r \\
j
\end{array}\right) B_{j} \frac{n^{r+1-j}}{r+1-j} & \text { if } r \geq 1, \\
n-1 & \text { if } r=0,\end{cases}
$$

where $r$ and $n$ are any non-negative integers satisfying $n \geq 2$.

The multiple zeta function can be represented as

$$
\zeta_{k}\left(s_{1}, \ldots, s_{k}\right)=\sum_{n_{k}=1}^{\infty} \frac{1}{n_{k}^{s_{k}}} \sum_{n_{k-1}=1}^{n_{k}-1} \frac{1}{n_{k-1}^{s_{k-1}}} \cdots \sum_{n_{2}=1}^{n_{3}-1} \frac{1}{n_{2}^{s_{2}}} \sum_{n_{1}=1}^{n_{2}-1} \frac{1}{n_{1}^{s_{1}}} .
$$

The region in which $\zeta_{k}\left(s_{1}, \ldots, s_{k}\right)$ is convergent when $\Re s_{i} \leq 0(i=1, \ldots, k-1)$ is calculated from the estimate

$$
\sum_{n \leq x} \frac{1}{n^{s}} \ll x^{1-\Re s} \quad \text { if } \Re s \leq 0,
$$

where $x$ is a real number satisfying $x \geq 1$. By (7.2) and (7.3), if $\Re s_{i} \leq 0$ $(i=1, \ldots, k-1)$, then $\zeta_{k}\left(s_{1}, \ldots, s_{k}\right)$ is absolutely convergent for

$$
\Re s_{k}>-\sum_{i=1}^{k-1} \Re s_{i}+k .
$$

Hence $\zeta_{k}\left(-r_{1}, \ldots,-r_{k-1}, s_{k}\right)$ is absolutely convergent for $\Re s_{k}>\sum_{j=1}^{k-1} r_{j}+k$. 
Then for $\Re s_{k}>\sum_{j=1}^{k-1} r_{j}+k$ we have

$$
\begin{aligned}
& \zeta_{k}\left(-r_{1}, \ldots,-r_{k-1}, s_{k}\right) \\
& = \begin{cases}\sum_{j=0}^{r_{1}}\left(\begin{array}{c}
r_{1} \\
j
\end{array}\right) \frac{B_{j}}{r_{1}+1-j} & \\
\times \zeta_{k-1}\left(-r_{1}-r_{2}-1+j,-r_{3}, \ldots,-r_{k-1}, s_{k}\right) & \text { if } r_{1}>0, \\
\zeta_{k-1}\left(-r_{2}-1,-r_{3}, \ldots,-r_{k-1}, s_{k}\right) & \\
-\zeta_{k-1}\left(-r_{2},-r_{3}, \ldots,-r_{k-1}, s_{k}\right) & \text { if } r_{1}=0\end{cases}
\end{aligned}
$$

from (7.1). In the case $k=2$, the above formula implies

$$
\zeta_{2}\left(-r_{1}, s_{2}\right)= \begin{cases}\sum_{j=0}^{r_{1}}\left(\begin{array}{c}
r_{1} \\
j
\end{array}\right) \frac{B_{j}}{r_{1}+1-j} \zeta\left(s_{2}-r_{1}-1+j\right) & \text { if } r_{1}>0 \\
\zeta\left(s_{2}-1\right)-\zeta\left(s_{2}\right) & \text { if } r_{1}=0 .\end{cases}
$$

Thus we have the meromorphic continuation of $\zeta_{2}\left(-r_{1}, s_{2}\right)$ to the whole $s_{2}$-plane. Further, the singularities of $\zeta_{2}\left(-r_{1}, s_{2}\right)$ are in $\Re s_{2}>0$ for $-r_{1} \in$ $\mathbb{Z}_{\leq 0}$. By using (7.4) and induction on $k$, we can obtain the meromorphic continuation of $\zeta_{k}\left(-r_{1}, \ldots,-r_{k-1}, s_{k}\right)$ and we can see that its singularities are in the half-plane $\Re s_{k}>0$. Hence we have

Lemma 7.3. The function $\zeta_{k}\left(-r_{1}, \ldots,-r_{k-1}, s_{k}\right)$ can be expressed by (7.4) for $\Re s_{k}>\sum_{j=1}^{k-1} r_{j}+k$. Then, inductively, (7.4) gives us the meromorphic continuation of $\zeta_{k}\left(-r_{1}, \ldots,-r_{k-1}, s_{k}\right)$ to the whole $s_{k}$-plane and the singularities of $\zeta_{k}\left(-r_{1}, \ldots,-r_{k-1}, s_{k}\right)$ are in the half-plane $\Re s_{k}>0$.

From Lemma 7.3, we may let $s_{k} \rightarrow-r_{k}$, where $r_{k}$ is a non-negative integer. Thus we have

THEOREM 7.4. Let $k$ be a positive integer satisfying $k \geq 3$. Then for any non-negative integers $r_{i}(i=1, \ldots, k)$, we have

$$
\zeta_{k}\left(\begin{array}{c}
1 \\
-r_{1},-r_{2}, \ldots,-r_{k}
\end{array}\right)=\zeta_{k}\left(\begin{array}{ccc}
\sigma(1) & \sigma(2) & \\
- & -r_{1}, & -r_{2}
\end{array}, \ldots,-r_{k}\right),
$$

where $\sigma \in \mathfrak{S}_{k}$ satisfies $\sigma(k)=k$.

8. An algorithm to calculate multiple zeta values for all coordinatewise limits. To end this article, we give an algorithm to calculate multiple zeta values for all coordinatewise limits at non-positive integers, which is described by induction on depth $k$. Namely, when calculating multiple zeta values of depth $k$, we assume that multiple zeta values for all coordinatewise limits of depth up to $k-1$ have already been obtained. Hereafter $\left(i_{1}, \ldots, i_{k}\right)$ indicates the order of the limiting process. Note that we can obtain multiple zeta values of depth $k$ for $i_{1}=1$ or $i_{k}=1$ by using Lemmas 3.1 and 3.2 , since $\zeta_{k}\left(-r_{1}, s_{2}, \ldots, s_{k}\right)$ is a finite sum of multiple zeta 
values of depth $k-1$ by Lemma 3.2. Similarly, the case of $i_{k}=1$ can be obtained from Lemma 3.1. Further, we showed that multiple zeta values for $i_{k}=k$ can be written using reverse values as in Theorem 7.4. Therefore we may focus only on the case $i_{1} \neq 1, i_{k} \neq 1$ and $i_{k} \neq k$.

Recall that Lemmas 3.1 and 3.2 give the analytic continuation of the multiple zeta function and the location of its singularities (1.1)-(1.3). Since indeterminacy points are special cases of singularity, we must observe carefully the behaviour of the multiple zeta function when one of the conditions (1.1)-(1.3) is satisfied; the case of (1.2) is particularly important.

8.1. The case $i_{k-1}>i_{k}>1$. From Lemma 3.1, we have

$$
\zeta_{k}\left(*, \ldots, *, s_{k-1}, \stackrel{i_{k}}{-r_{k}}\right)=\sum_{q=-1}^{r_{k}}\left(-r_{k}\right)_{q}^{+} a_{q} \zeta_{k-1}\left(*, \ldots, *, s_{k-1}-r_{k}+q\right) .
$$

Here the symbol $*$ stands for $\stackrel{-i_{\lambda}}{-} r_{\lambda}$ or $s_{\lambda}$ for $1 \leq \lambda \leq k-2$. We note that in the above formula condition (1.2), and hence of course (1.3), is not satisfied in general. Since the right-hand side above is a finite sum of multiple zeta values of depth $k-1$, we can calculate multiple zeta values for this case inductively. Therefore multiple zeta values for limiting processes of this type are described by multiple zeta values of depth $k-1$ at non-positive integers.

8.2. The case $i_{k-1}<i_{k}<k$. In this case, we have to consider the behaviour of $\zeta_{k}\left(s_{1}, \ldots,-r_{k-1}, s_{k}\right)$ at $s_{k}=r_{k} \in \mathbb{Z}$. The following lemma is an essential tool in this case. Hereafter we omit the index $\left(i_{1}, \ldots, i_{k}\right)$ for convenience.

LEMMA 8.1. $\zeta_{k}\left(s_{1}, \ldots, s_{k}\right)$ has a simple pole at $s_{k}=1$ with residue $\zeta_{k-1}\left(s_{1}, \ldots, s_{k-1}\right)$ for fixed $\left(s_{1}, \ldots, s_{k-1}\right) \in \mathbb{C}^{k-1}$ which does not satisfy the conditions (1.2) and (1.3) with $s_{k}=1$. Further, let $r_{j}(j=v, \ldots, k-1)$ be a non-negative integer, and set

$$
R_{k, v}:=\sum_{j=v}^{k} r_{j}
$$

and

$$
Z_{k, v}\left(s_{k}\right):=\zeta_{k}\left(s_{1}, \ldots, s_{v-1},-r_{v}, \ldots,-r_{k-1}, s_{k}\right)
$$

$(2 \leq v \leq k-1$ for $k \geq 3)$, where $\left(s_{1}, \ldots, s_{v-1},-r_{v}, \ldots,-r_{k-1}\right) \in \mathbb{C}^{k-1}$ does not satisfy condition (1.3) for $k-v+2 \leq j \leq k$ with $s_{k}=r_{k} \in \mathbb{Z}$ and is fixed. Then $Z_{k, v}\left(s_{k}\right)$ has a simple pole at each $s_{k}=r_{k} \in \mathbb{Z}_{\geq 1}$ for $1 \leq r_{k} \leq(k-v)+R_{k-1, v}+1$ with residue $C_{k, v}\left(s_{1}, \ldots,-r_{k-1} ; r_{k}\right)$ which satisfies the following inductive relation: 


$$
\begin{aligned}
C_{k, v}\left(s_{1}, \ldots, s_{v-1},-r_{v}, \ldots,-r_{k-1} ; r_{k}\right) & \\
= & \delta_{r_{k}} \zeta_{k-1}\left(s_{1}, \ldots, s_{v-1},-r_{v}, \ldots,-r_{k-1}\right) \\
& +\sum_{q=M_{k,\left(\delta_{r_{k}}-1\right)}}^{(k-v)+R_{k-1, v}-r_{k}}\left(r_{k}\right)_{q}^{+} a_{q} \\
& \times C_{k-1, v}\left(s_{1}, \ldots, s_{v-1},-r_{v}, \ldots,-r_{k-2} ;-r_{k-1}+r_{k}+q\right),
\end{aligned}
$$

where $M_{k, \nu}=M_{k, \nu}\left(-r_{k-1} ; r_{k}\right):=\max \left\{\nu, 1-\left(r_{k}-r_{k-1}\right)\right\}$ and

$$
\delta_{\mu}= \begin{cases}1 & \text { if } \mu=1, \\ 0 & \text { otherwise. }\end{cases}
$$

REMARK 8.2. The above lemma still holds if some (not all) of $s_{1}, \ldots, s_{v-1}$ are fixed non-positive integers. Namely, the integer $v$ in the above lemma implies that $v:=1+\max \left\{m \in \mathbb{N}: i_{m}>i_{k}\right\}$ for a given order of the limiting process $\left(i_{1}, \ldots, i_{k}\right)$.

Proof of Lemma 8.1. First, we prove the first assertion of the above lemma. Hereafter we assume that $l$ is a sufficiently large positive integer. From Lemma 3.1,

$$
\begin{aligned}
& \zeta_{k}\left(s_{1}, \ldots, s_{k}\right)=\frac{\zeta_{k-1}\left(s_{1}, \ldots, s_{k-1}+s_{k}-1\right)}{s_{k}-1} \\
& \quad+\sum_{q=0}^{l}\left(s_{k}\right)_{q}^{+} a_{q} \zeta_{k-1}\left(s_{1}, \ldots, s_{k-1}+s_{k}+q\right)-\sum_{0<n_{1}<\cdots<n_{k-1}} \frac{\phi_{l}\left(n_{k-1}, s_{k}\right)}{n_{1}^{s_{1}} \cdots n_{k-1}^{s_{k-1}}} .
\end{aligned}
$$

The possible singularities of $\zeta_{k}\left(s_{1}, \ldots, s_{k}\right)$ come from the factor $1 /\left(s_{k}-1\right)$ in the first term and from $\zeta_{k-1}\left(s_{1}, \ldots, s_{k-1}+s_{k}+q\right)$ 's. The former gives (1.1), while the latter give (1.2) and (1.3). Therefore $\zeta_{k-1}\left(s_{1}, \ldots, s_{k}+s_{k-1}+q\right)$ 's are regular for $s_{k}=1$ and $\left(s_{1}, \ldots, s_{k-1}, 1\right) \in \mathbb{C}^{k}$ which do not satisfy conditions (1.2) and (1.3). Hence for fixed such $\left(s_{1}, \ldots, s_{k-1}\right)$ we have

$$
\zeta_{k}\left(s_{1}, \ldots, s_{k}\right) \sim \frac{\zeta_{k-1}\left(s_{1}, \ldots, s_{k-1}\right)}{s_{k}-1} \quad \text { as } s_{k} \rightarrow 1 .
$$

Here we have used the Taylor expansion of $\zeta_{k-1}\left(s_{1}, \ldots, s_{k-1}+s_{k}-1\right)$ at $s_{k}=1$.

Secondly, we prove the second assertion of Lemma 8.1. The proof is by induction on $k$. When $k=3$, we have

$$
\begin{aligned}
\zeta_{3}\left(s_{1},-r_{2}, s_{3}\right)= & \frac{\zeta_{2}\left(s_{1}, s_{3}-r_{2}-1\right)}{s_{3}-1} \\
& +\sum_{q=0}^{l}\left(s_{3}\right)_{q}^{+} a_{q} \zeta_{2}\left(s_{1}, s_{3}-r_{2}+q\right)-\sum_{0<n_{1}<n_{2}} \frac{\phi_{l}\left(n_{2}, s_{3}\right)}{n_{1}^{s_{1}} n_{2}^{-r_{2}}} .
\end{aligned}
$$


From condition (1.2), $\zeta_{3}\left(s_{1},-r_{2}, s_{3}\right)$ is regular for $s_{3}=r_{3} \geq r_{2}+3$. Further, we can see that $\zeta_{3}\left(s_{1},-r_{2}, s_{3}\right)$ is regular at $s_{3}=-r_{3} \in \mathbb{Z}_{\leq 0}$. In fact, $\zeta_{2}\left(s_{1}, s_{3}-r_{2}+q\right)$ has a simple pole at $s_{3}=-r_{3}$ when $q=r_{2}+r_{3}+1$. However, $\left(s_{3}\right)_{r_{2}+r_{3}+1}^{+}$also has a simple zero at $s_{3}=-r_{3}$. Hence those cancel each other. Therefore we may focus on the case $s_{3}=r_{3} \in \mathbb{Z}_{\geq 1}$ and $1 \leq r_{3} \leq r_{2}+2$. First we consider the case $r_{3} \geq 2$. We assume that $\left(s_{1},-r_{2}, r_{3}\right) \in \mathbb{C}^{3}$ does not satisfy condition (1.3) and $s_{1}$ is fixed. By the first assertion of Lemma 8.1, $\zeta_{2}\left(s_{1}, s_{3}-r_{2}+q\right)$ has a simple pole at $s_{3}=r_{3}$ when $q=r_{2}-r_{3}+1$, and its residue is $\zeta\left(s_{1}\right)$. For $r_{3}=1$, the first term on the right-hand side of the above formula is also the singular part, and its residue is $\zeta_{2}\left(s_{1},-r_{2}\right)$. Hence we have

$$
\begin{aligned}
& \zeta_{3}\left(s_{1},-r_{2}, s_{3}\right) \\
& \sim \frac{1}{s_{3}-r_{3}}\left\{\delta_{r_{3}} \zeta_{2}\left(s_{1},-r_{2}\right)+\left(r_{3}\right)_{r_{2}-r_{3}+1}^{+} a_{r_{2}-r_{3}+1} \zeta\left(s_{1}\right)\right\} \quad \text { as } s_{3} \rightarrow r_{3} .
\end{aligned}
$$

Note that the residue of the above formula equals $C_{3,2}\left(s_{1},-r_{2} ; r_{3}\right)$ of Lemma 8.1.

Next we assume that the second assertion of Lemma 8.1 is true up to $k-1$. Similarly, from Lemma 3.1, we have

$$
\begin{aligned}
Z_{k, v}\left(s_{k}\right)= & \frac{\zeta_{k-1}\left(s_{1}, \ldots, s_{v-1},-r_{v}, \ldots, s_{k}-r_{k-1}-1\right)}{s_{k}-1} \\
& +\sum_{q=0}^{l}\left(s_{k}\right)_{q}^{+} a_{q} \zeta_{k-1}\left(s_{1}, \ldots, s_{v-1},-r_{v}, \ldots, s_{k}-r_{k-1}+q\right) \\
& -\sum_{0<n_{1}<\cdots<n_{k-1}} \frac{\phi_{l}\left(n_{k-1}, s_{k}\right)}{n_{1}^{s_{1}} \cdots n_{k-1}^{-r_{k-1}}}
\end{aligned}
$$

It is easily seen that $Z_{k, v}\left(s_{k}\right)$ is regular at $s_{k}=-r_{k} \in \mathbb{Z}_{\leq 0}$ for the same reason as in the case of depth 3. Further, from (1.3) for $j=k-v+1, Z_{k, v}\left(s_{k}\right)$ is regular at $s_{k}=r_{k} \in \mathbb{Z}_{>1}$ for $r_{k} \geq(k-v)+R_{k-1, v}+2$. Hence we may consider only the case $s_{k}=r_{k} \in \mathbb{Z}_{\geq 1}$ and $1 \leq r_{k} \leq(k-v)+R_{k-1, v}+1$. First, we consider the case $r_{k} \geq 2$. Then from the inductive assumption and (8.4), each $\zeta_{k-1}\left(s_{1}, \ldots,-r_{k-2}, s_{k}-r_{k-1}+q\right)$ for $M_{k,-1} \leq q \leq(k-v)+R_{k-1, v}-r_{k}$ has a simple pole at $s_{k}=r_{k}$ with residue $C_{k-1, v}\left(s_{1}, \ldots,-r_{k-2} ; r_{k}-r_{k-1}+q\right)$. Thus we obtain

$$
\begin{aligned}
Z_{k, v}\left(s_{k}\right) \sim & \frac{1}{s_{k}-r_{k}}\left\{\sum_{q=M_{k,-1}}^{(k-v)+R_{k-1, v}-r_{k}}\left(r_{k}\right)_{q}^{+} a_{q}\right. \\
& \left.\times C_{k-1, v}\left(s_{1}, \ldots,-r_{v}, \ldots,-r_{k-2} ; r_{k}-r_{k-1}+q\right)\right\} \quad \text { as } s_{k} \rightarrow r_{k} .
\end{aligned}
$$


On the other hand, when $r_{k}=1$, the first term on the right-hand side of (8.4) is also the singular part with residue $\zeta_{k-1}\left(s_{1}, \ldots,-r_{v}, \ldots,-r_{k}\right)$. Hence we have the second assertion of Lemma 8.1.

We return to the case $i_{k-1}<i_{k}<k$. From Lemma 3.1, we have

$$
\begin{aligned}
\zeta_{k}\left(s_{1}, \ldots,-r_{v}, \ldots,\right. & \left.-r_{k-1}, s_{k}\right) \\
= & \sum_{q=-1}^{l}\left(s_{k}\right)_{q}^{+} a_{q} \zeta_{k-1}\left(s_{1}, \ldots,-r_{v}, \ldots, s_{k}-r_{k-1}+q\right) \\
& -\sum_{0<n_{1}<\cdots<n_{k-1}} \frac{\phi_{l}\left(n_{k-1}, s_{k}\right)}{n_{1}^{s_{1}} \cdots n_{k-1}^{-r_{k-1}}} .
\end{aligned}
$$

If we let $s_{k} \rightarrow-r_{k}$, then Lemma 8.1 suggests that there exist singular terms in the range $1+R_{k, k-1} \leq q \leq(k-v)+R_{k, v}$. However, the factor $\left(s_{k}\right)_{q}^{+}$also has a simple zero at $z_{k}=-r_{k}$. Hence

$$
\begin{aligned}
& \lim _{s_{k} \rightarrow-r_{k}} \sum_{q=1+R_{k, k-1}}^{(k-v)+R_{k, v}}\left(s_{k}\right)_{q}^{+} a_{q} \zeta_{k-1}\left(s_{1}, \ldots,-r_{v}, \ldots,-r_{k-2}, s_{k}-r_{k-1}+q\right) \\
& =\lim _{s_{k} \rightarrow-r_{k}} \sum_{l=1}^{(k-v)+R_{k-2, v}}\left(s_{k}\right)_{l+R_{k, k-1}}^{+} a_{l+R_{k, k-1}} \\
& \times \zeta_{k-1}\left(s_{1}, \ldots,-r_{v}, \ldots,-r_{k-2}, s_{k}+r_{k}+l\right) \\
& =\sum_{l=1}^{(k-v)+R_{k-2, v}}(-1)^{r_{k}} r_{k} !\left(r_{k-1}+l-1\right) ! a_{l+R_{k, k-1}} \\
& \times C_{k-1, v}\left(s_{1}, \ldots,-r_{v}, \ldots,-r_{k-2} ; l\right) .
\end{aligned}
$$

Thus we obtain

$$
\begin{aligned}
\zeta_{k}\left(s_{1}, \ldots,-r_{v}, \ldots,-r_{k-1},-r_{k}\right) & \\
= & \sum_{q=-1}^{r_{k}}\left(-r_{k}\right)_{q}^{+} a_{q} \zeta_{k-1}\left(s_{1}, \ldots,-r_{v}, \ldots,-r_{k}-r_{k-1}+q\right) \\
& +\sum_{l=1}^{(k-v)+R_{k-2, v}}(-1)^{r_{k}} r_{k} !\left(r_{k-1}+l-1\right) ! a_{l+R_{k, k-1}} \\
& \times C_{k-1, v}\left(s_{1}, \ldots,-r_{v}, \ldots,-r_{k-2} ; l\right) .
\end{aligned}
$$

Hence the multiple zeta function of depth $k$ can also be expressed by those of depth $\leq k-1$ in this case. Therefore, when $i_{k-1}<i_{k}<k$, we can obtain multiple zeta values for coordinatewise limits at non-positive integers inductively by using (8.5). 
REMARK 8.3. Note that the residue $C_{k, v}$ involves the multiple zeta values of depth $\leq k-1$ (for instance see (8.3)). Therefore the assumption on variables in Lemma 8.1 is required to avoid singularities of multiple zeta functions. However, in the case of the limiting process with order, this assumption may be removed once we apply Lemma 8.1.

EXAMPLE 8.4. When $k=4$ and $\left(i_{1}, i_{2}, i_{3}, i_{4}\right)=(4,2,1,3)$, we put $v=2$ in Lemma 8.1. Then

$$
\begin{aligned}
\zeta_{4}\left(-r_{1},-r_{2},\right. & 1 \\
- & \left.r_{3},-r_{4}\right)=\sum_{q=-1}^{r_{4}}\left(-r_{4}\right)_{q}^{+} a_{q} \zeta_{3}\left(\stackrel{3}{3} \stackrel{1}{1},-r_{2},-r_{3}-r_{4}^{2}+q\right) \\
& +(-1)^{r_{4}} r_{4} ! r_{3} ! a_{1+r_{3}+r_{4}} \zeta_{2}\left(-r_{1}^{2},-r_{2}\right) \\
& +(-1)^{r_{4}} r_{4} ! \sum_{1 \leq l \leq r_{2}+1}\left(r_{3}+l\right) ! a_{r_{3}+r_{4}+1+l}(l+1)_{r_{2}-l}^{+} a_{r_{2}-l} \zeta\left(-r_{1}\right) .
\end{aligned}
$$

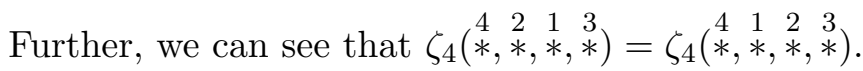

When $\left(i_{1}, i_{2}, i_{3}, i_{4}\right)=(2,4,1,3)$, we have

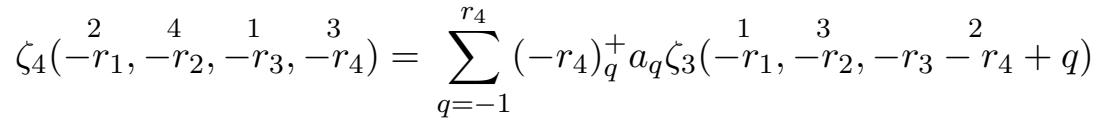

$$
\begin{aligned}
& +(-1)^{r_{4}} r_{4} ! r_{3} ! a_{r_{4}+r_{3}+1} \zeta_{2}\left(\stackrel{1}{-} r_{1},-r_{2}\right) .
\end{aligned}
$$

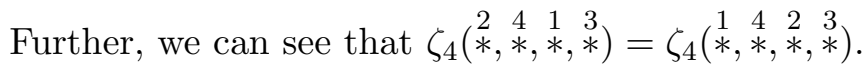

When $\left(i_{1}, i_{2}, i_{3}, i_{4}\right)=(4,3,1,2)$, we have

$$
\begin{aligned}
\zeta_{4}\left(\stackrel{4}{-} r_{1}, \stackrel{3}{-} r_{2},-r_{3}, \stackrel{2}{-} r_{4}\right)= & \sum_{q=-1}^{r_{4}}\left(-r_{4}\right)+\underset{q}{+} a_{q} \zeta_{3}\left(\stackrel{3}{-} r_{1}, \stackrel{2}{-} r_{2},-r_{3}-{ }^{-} r_{4}+q\right) \\
& +(-1)^{r_{4}} r_{4} ! r_{3} ! a_{r_{4}+r_{3}+1} \zeta_{2}\left(\stackrel{2}{-} r_{1},-r_{2}\right) .
\end{aligned}
$$

When $\left(i_{1}, i_{2}, i_{3}, i_{4}\right)=(3,4,1,2)$, we have

$$
\begin{aligned}
\zeta_{4}\left(\stackrel{3}{-} r_{1},-\stackrel{4}{r}_{2}, \stackrel{1}{-} r_{3}, \stackrel{2}{-} r_{4}\right)= & \sum_{q=-1}^{r_{4}}\left(-r_{4}\right)_{q}^{+} a_{q} \zeta_{3}\left(\stackrel{2}{-} r_{1}, \stackrel{3}{-} r_{2},-r_{3}-{ }^{1} r_{4}+q\right) \\
& +(-1)^{r_{4}} r_{4} ! r_{3} ! a_{r_{4}+r_{3}+1} \zeta_{2}\left(\stackrel{1}{-} r_{1},-r_{2}\right) .
\end{aligned}
$$

Acknowledgements. The author would like to express his gratitude to Professor Kohji Matsumoto and Professor Yoshio Tanigawa for valuable advice and comments. Thanks are also due to Professor Yasushi Komori for his helpful suggestions. 


\section{References}

[1] S. Akiyama, S. Egami and Y. Tanigawa, Analytic continuation of multiple zetafunctions and their values at non-positive integers, Acta Arith. 98 (2001), 107-116.

[2] S. Akiyama and Y. Tanigawa, Multiple zeta values at non-positive integers, Ramanujan J. 5 (2001), 327-351.

[3] F. V. Atkinson, The mean-value of the Riemann zeta function, Acta Math. 81 (1949), 353-376.

[4] L. Euler, Meditationes circa singulare serierum genus, Novi Comm. Acad. Sci. Petropol 20 (1775), 140-186, reprinted in Opera Omnia ser. I, vol. 15, B. G. Teubner, Berlin, 1927, 217-267.

[5] A. Granville, A decomposition of Riemann's zeta-function, in: London Math. Soc. Lecture Note Ser. 247, Cambridge Univ. Press, 1997, 95-101.

[6] M. Hoffman, Multiple harmonic series, Pacific J. Math. 152 (1992), 275-290.

[7] K. Kamano, The multiple Hurwitz zeta-function and generalization of Lerch's formula, Tokyo J. Math. 29 (2006), 61-73.

[8] Y. Komori, An integral representation of multiple Hurwitz-Lerch zeta function and generalized multiple Bernoulli numbers, Ramanujan J., to appear.

[9] K. Matsumoto, The analytic continuation and the asymptotic behaviour of certain multiple zeta-functions I, J. Number Theory 101 (2003), 223-243.

[10] —, Functional equations for double zeta-functions, Math. Proc. Cambridge Philos. Soc. 136 (2004), 1-7.

[11] Y. Ohno, A generalization of the duality and sum formulas on the multiple zeta values, J. Number Theory 74 (1999), 39-43.

[12] J. Q. Zhao, Analytic continuation of multiple zeta functions, Proc. Amer. Math. Soc. 128 (2000), 1275-1283.

Graduate School of Mathematics

Nagoya University

Chikusa-ku, Nagoya 464-8602, Japan

E-mail: d06002n@math.nagoya-u.ac.jp

Received on 9.11.2007

and in revised form on 25.9.2008 